
\title{
$\begin{array}{ll}\text { Research Square } & \begin{array}{l}\text { Preprints are preliminary reports that have not undergone peer review. } \\ \text { They should not be considered conclusive, used to inform clinical practice, } \\ \text { or referenced by the media as validated information. }\end{array}\end{array}$
}

\section{Clinical course and outcomes of secondary bloodstream infection patients with COVID-19 in Wuhan, China: A retrospective study}

\section{Jie Li}

Department of Pharmacy, Union Hospital, Tongji Medical College, Huazhong University of Science and Technology Junwei Wang

Department of Pharmacy, Union Hospital, Tongji Medical College, Huazhong University of Science and Technology

Yi Yang

Department of Pharmacy, Union Hospital, Tongji Medical College, Huazhong University of Science and Technology

Peishan Cai

Department of Pharmacy, Union Hospital, Tongji Medical College, Huazhong University of Science and Technology

\section{Jingchao Cao}

Department of Pharmacy, Union Hospital, Tongji Medical College, Huazhong University of Science and Technology

\section{Sanlan Wu}

Department of Pharmacy, Union Hospital, Tongji Medical College, Huazhong University of Science and Technology

\section{Yu Zhang}

Department of Pharmacy, Union Hospital, Tongji Medical College, Huazhong University of Science and Technology

Xuefeng Cai ( $\nabla$ caiwhxh@163.com)

Union Hospital, Tongji Medical College, Huazhong University of Science and Technology https://orcid.org/0000-0003-2572-2950

\section{Research}

Keywords: secondary bloodstream infection, COVID-19, mortality, clinical course, outcomes

Posted Date: May 18th, 2021

DOI: https://doi.org/10.21203/rs.3.rs-530370/v1

License: (1) This work is licensed under a Creative Commons Attribution 4.0 International License. Read Full License 


\section{Abstract \\ Background}

Secondary bloodstream infection (SBI) is considered one of the most perilous complications of coronavirus disease 2019 (COVID-19). Although the etiology of SBI has been reported, the clinical course and outcomes of this illness have not been well described.

\section{Methods}

In this retrospective study, all patients with confirmed SBI were included from a cohort of 1,651 patients hospitalized with COVID-19 in the Wuhan Union Hospital between January 25 and April 23, 2020. Demographics, clinical features, laboratory findings, treatments and outcomes were extracted from electronic medical records. The data were compared between survivors and non-survivors based on whether patients died within 30 days following SBI onset.

\section{Results}

A total of 31 patients corresponding to $1.9 \%(31 / 1,651)$ who exhibited SBI were included in the present study. The median time from admission to the onset of SBI was 22.0 days (2.0-64.0). The most common symptoms were shortness of breath (74\%), fever (65\%) and decreased blood pressure (52\%) at the onset of SBI. The levels of white blood cell count, C-reactive protein, and procalcitonin were mostly elevated. Carbapenem-resistant Klebsiella pneumoniae (15 [48\%]) and Acinetobacter baumannii (7 [23\%]) were the main pathogens. In empirical treatment, carbapenems were still the first choice. The 30-day mortality following SBI onset and the hospital mortality of the 31 patients were $67.7 \%(21 / 31)$ and $77.4 \%(24 / 31)$, respectively. Almost every patient with SBI had complication due to sepsis (28 [90\%]). The median time periods to sepsis and septic shock were 1 days $(1.0 \sim 5.0)$ and 3.0 days (1.0 12.0), respectively. The proportion of carbapenem-resistant gram-negative bacteria (CRGNB) detected in blood cultures of non-survivors was higher (18 [86\%] vs. 4 [40\%]) compared to that of the survivors. These patients were more likely to develop sepsis (21 [100\%] vs. 7 [70\%]), and acute cardiac injury (19 $[90 \%]$ vs. $5[50 \%])$.

\section{Conclusions}

The mortality of SBI patients with COVID-19 is considerable. Increased risk of mortality was noted for the following three subgroups: Patients infected with CRGNB, patients with complications of sepsis and patients with acute cardiac injury.

\section{Introduction}

The global pandemic of coronavirus disease 2019 (COVID-19) is a dominant event in 2020, infecting more than 146 million people and being responsible for more than 3.0 million fatalities.[1, 2] Due to the lack of effective severe acute respiratory syndrome coronavirus 2 (SARS-CoV2) antiviral therapy, active prevention and treatment of complications is still one of the main measures for hospitalized patients with COVID19, notably for severe ill patients.[3] Secondary infection is one of the main complications of COVID-19. [4-6]According to this report, [5] half of the non-survivors experienced a secondary infection. For one type, although the incidence of secondary bloodstream infection (SBI) was estimated from $1.6 \%$ to $5.6 \%,[7-10]$ its mortality rate was relatively higher. Due to this evidence, it is very important to study the clinical course of $\mathrm{SBI}$ and its treatment strategies. Previous studies have described the etiology and risk factors of $\mathrm{SBI}$ using specific retrospective analysis, although the clinical course and outcomes of SBI have not been well described. $[7,8,10,11]$ The present study aimed to review the entire clinical course and outcomes of SBI with COVID-19 and discover predictors of death from SBI and possible effective treatment options, which will be of considerable value for reducing the mortality of this disease.

\section{Methods}

\section{Study design and participants}

This retrospective study was performed at the West Campus of Wuhan Union Hospital. From January 25 to April 23 2020, the function of this hospital was only as a designated hospital for the treatment of COVID-19. During this period, a total of 1,651 patients who were diagnosed with COVID-19 according to Chinese management guidelines for COVID-19 were treated here and included in our study.[12] 
The present study was approved by the Ethics Committee of Union Hospital, Tongji Medical College, Huazhong University of Science and Technology (Permission number: [2020]0104). Written informed consent was waived due to the rapid emergence of this infectious disease.

\section{Data collection}

We reviewed the etiological results of all 1,651 COVID-19 patients in the laboratory examination system and screened patients with positive blood culture results. The data of these patients were extracted from electronic medical records and nursing records, including demographics, clinical features, laboratory findings, treatments and outcomes. If an organism cultured from blood samples was not a recognized pathogen, clinical symptoms (fever, chills, or hypotension) that were not related to infections at another site were observed. Concomitantly, it was observed that the organism was cultured from 2 or more blood cultures drawn on separate occasions. When the two conditions could not be met at the same time, the organism was considered a skin contaminant and was excluded from the study.[13] When the patients had multiple secondary bloodstream infections (SBIs) during hospitalization, the first SBI was included in the study. The data including oxygenation index, platelets, bilirubin, cardiovascular indices, Glasgow Coma Scale score, creatinine and urine output were collected at admission and at onset of SBI to calculate the Sequential Organ Failure Assessment (SOFA) score.[14] The data were compared between survivors and non-survivors according to whether patients did not survive within 30 days following SBI onset.

All data were checked by two physicians (JL and JW) and a third researcher (YY) adjudicated any difference in interpretation between the two primary reviewers.

\section{Definitions}

SBI was diagnosed according to the CDC/NHSN surveillance definition of health care-associated infection and occurred during hospitalization due to COVID-19.[13] The onset time of SBI was defined as the date when the positive blood culture was collected. The illness severity of COVID-19 at admission was classified according to the Chinese management guidelines for COVID-19 (version 7.0).[12] Multidrugresistant (MDR) was defined according to an international expert proposal formulated by the European Centre for Disease Prevention and Control (ECDC) and the Centers for Disease Control and Prevention (CDC).[15] Therapy with antimicrobial drugs prior to and after the availability of the susceptibility results was defined as empirical antimicrobial therapy and targeted therapy, respectively.[16] Fever was defined as axillary temperature of at least $37.3^{\circ} \mathrm{C}$. Sepsis and septic shock were diagnosed according to the Third International Consensus Definitions for Sepsis and Septic Shock.[14] Acute kidney injury was diagnosed according to the KDIGO clinical practice guideline for acute kidney injury [17]and acute liver injury was diagnosed if alanine aminotransferase and/or aspartate aminotransferase was increased higher than 5 times of the upper normal limit. Acute cardiac injury was diagnosed if there was a rise and/or fall in cardiac troponin concentrations exceeding the biological and/or analytical variation.[18] Coagulopathy was defined as a 3-second extension of pro thrombin time or a 5second extension of activated partial thromboplastin time.

\section{Statistical analysis}

Continuous and categorical variables are presented as median (IQR) and n (\%), respectively. The Mann-Whitney $U$ test, $\chi^{2}$ test, or Fisher's exact tests were used to compare differences between survivors and non-survivors where appropriate. A P-value $<0.05$ was considered for significant differences. All statistical analyses were performed by IBM SPSS Statistics 26.0.

\section{Results}

By April 23, 2020, a total of 39 patients exhibited at least one positive blood culture result during hospitalization. The blood cultures of 8 patients were confirmed to be contaminated by skin symbiotic bacteria. Therefore, these patients were not considered experienced SBI cases. A total of 31 patients (1.9\%) with SBI were included in the present study. The median age of the patients was 67.0 years (IQR $38.0 \sim 80.0$ ), 25 $(81 \%)$ of whom were men (Table 1). The majority of the patients had underlying diseases (26 [84\%]) and the median of Charlson Comorbidity Index (CCI) at admission was 2.0 (IQR $0 \sim 5.0$ ). At admission, 8 patients were assessed as severely ill, while 23 patients were assessed as critically ill. The median of SOFA score was 4.0 (IQR $1.0 \sim 9.0$ ) and the majority of the patients exhibited poor oxygenation. The median duration from admission to SBI onset was 22.0 days (2.0-64.0). The mortality within 30 days following SBI onset and the hospital mortality of the 31 patients were $67.7 \%(21 / 31)$ and $77.4 \%$ (24/31), respectively. 
Table 1

Demographic and clinical findings of patients at admission

\begin{tabular}{|c|c|c|c|c|}
\hline & $\begin{array}{l}\text { Total } \\
(n=31)\end{array}$ & $\begin{array}{l}\text { Non-survivor } \\
(n=21)\end{array}$ & $\begin{array}{l}\text { Survivor } \\
(n=10)\end{array}$ & p value \\
\hline Ages, years & $67.0(38.0 \sim 80.0)$ & $67.0(48.0 \sim 79.0)$ & $72.0(38.0 \sim 80.0)$ & 0.724 \\
\hline Sex & & & & 1.000 \\
\hline Female & $6(19 \%)$ & $4(19 \%)$ & $2(20 \%)$ & $\ldots$ \\
\hline Male & $25(81 \%)$ & $17(81 \%)$ & $8(80 \%)$ & $\cdots$ \\
\hline Comorbidity & $26(84 \%)$ & $17(81 \%)$ & $9(90 \%)$ & 1.000 \\
\hline Hypertension & $16(52 \%)$ & $10(48 \%)$ & $6(60 \%)$ & 0.700 \\
\hline Diabetes & $3(10 \%)$ & $2(10 \%)$ & $1(10 \%)$ & 1.000 \\
\hline Coronary heart disease & $10(32 \%)$ & $8(38 \%)$ & $2(20 \%)$ & 0.430 \\
\hline Coronary lung disease & $4(13 \%)$ & $4(19 \%)$ & 0 & 0.280 \\
\hline Chronic kidney disease & $1(3 \%)$ & $1(5 \%)$ & 0 & 1.000 \\
\hline Carcinoma & $2(6 \%)$ & $1(5 \%)$ & $1(10 \%)$ & 1.000 \\
\hline $\mathrm{CCl}$ & $2(0 \sim 5.0)$ & $2.0(0 \sim 5.0)$ & $1.5(0 \sim 4.0)$ & 0.693 \\
\hline SOFA score & $4.0(1.0 \sim 9.0)$ & $4.0(3.0 \sim 9.0)$ & $4.0(1.0 \sim 6.0)$ & 0.950 \\
\hline Disease severity status & & & & 0.074 \\
\hline Severe & $8(26 \%)$ & $3(14 \%)$ & $5(50 \%)$ & $\cdots$ \\
\hline Critical & $23(74 \%)$ & $18(86 \%)$ & $5(50 \%)$ & $\ldots$ \\
\hline
\end{tabular}

The most common symptoms were shortness of breath (74\%), fever (65\%), decreased blood pressure (52\%; Table 2) following SBI. Since the majority of the patients were provided sedative measures prior to the onset of SBI, the manifestations of chills were rarely observed. 
Table 2

Clinical features and laboratory findings of patients at SBI onset

\section{Total $(\mathbf{n}=\mathbf{3 1})$ \\ Non-survivor $(\mathrm{n}=$}

\section{Clinical features}

Fever

Highest temperature, ${ }^{\circ} \mathrm{C}$

$>39.0$

$38.1 \sim 39.0$

$37.3 \sim 38.0$

$<37.3$

Fear of cold

Chills

Decreased blood pressure

Shortness of breath

Change of consciousness

Sedation

SOFA score

\section{Laboratory findings}

White blood cell count, $\times 108 / L$

$<4$

$\geq 4$ to $\leq 10$

$>10$ to $<18$

$\geq 18$

Neutrophil count, $\times 10 \otimes / L$

$<1.8$

$1.8 \sim 6.3$

$>6.3$

Lymphocyte count, $\times 10 \otimes / L$

$<1.1$

$1.1 \sim 3.2$

C reactive protein, $\mathrm{mg} / \mathrm{L}$

$<8$

$\geq 8$ to $<50$

$\geq 50$ to $<100$

$\geq 100$

Procalcitonin, $\mathrm{ng} / \mathrm{mL}$

$\geq 0.1$ to $<0.25$

$20(65 \%)$

$8(26 \%)$

$8(26 \%)$

$4(13 \%)$

$11(35 \%)$

$2(6 \%)$

$2(6 \%)$

$16(52 \%)$

$23(74 \%)$

$11(35 \%)$

$26(84 \%)$

$8.0(2.0 \sim 19.0)$

$12.5(0.09 \sim 39.26)$

$4(13 \%)$

5 (16\%)

17 (55\%)

5 (16\%)

$11.1(0.02 \sim 37.73)$

$2(6 \%)$

5 (16\%)

$24(77 \%)$

$0.5(0.06 \sim 1.6)$

$29(94 \%)$

2 (6\%)

115.9 (2.41

193.13)

$3(10 \%)$

$3(10 \%)$

$8(26 \%)$

17 (55\%)

$1.1(0.14 \sim 44.62)$

$4(13 \%)$

The data represent median (IQR) or $n(\%)$. The $p$ values were calc
appropriate. The ${ }^{*} \chi^{2}$ test was used to compare all subcategories.
21)

$13(62 \%)$

$7(70 \%)$

1.000

Survivor $(n=10)$

value

\begin{tabular}{lll}
\hline $6(29 \%)$ & $2(20 \%)$ & $0.722^{*}$ \\
$4(19 \%)$ & $4(40 \%)$ &.. \\
\hline $3(14 \%)$ & $1(10 \%)$ &.. \\
\hline $8(38 \%)$ & $3(30 \%)$ &.. \\
\hline 0 & $2(20 \%)$ & 0.097 \\
\hline 0 & $2(20 \%)$ & 0.097 \\
$10(48 \%)$ & $6(60 \%)$ & 0.704 \\
$18(86 \%)$ & $5(50 \%)$ & 0.074 \\
$10(48 \%)$ & $1(10 \%)$ & 0.055 \\
$19(90 \%)$ & $7(70 \%)$ & 0.300 \\
$8.0(3.0 \sim 19.0)$ & $6.0(2.0 \sim 12.0)$ & 0.233
\end{tabular}

$12.5(0.09 \sim 39.26)$

$12.6(5.56 \sim 28.8)$

0.574

$4(19 \%)$

$0(0 \%)$

0.360 *

$3(14 \%)$

$2(20 \%)$

12 (57\%)

$5(50 \%)$

$3(30 \%)$

11.5 (5.54

27.91)

.519

$11.1(0.02 \sim 37.73)$

0

$1.000 *$

$2(20 \%)$

$8(80 \%)$

$16(76 \%)$

$0.6(0.15 \sim 1.12)$

0.466

$0.5(0.06 \sim 1.6)$

$9(90 \%)$

$1.000 *$

1 (10\%)

62.4 (2.82

192.85)

119.2 (2.41

193.13)

2 (20\%)

1 (5\%)

$1(10 \%)$

$2(10 \%)$

$3(30 \%)$

$5(24 \%)$

$4(40 \%)$

$13(62 \%)$

$4(40 \%)$

$1.4(0.14 \sim 44.62)$

$0.6(0.16 \sim 32.5)$

0.159

$2(10 \%)$

$2(20 \%)$

0.424 * 


\begin{tabular}{|c|c|c|c|c|}
\hline & Total $(n=31)$ & $\begin{array}{l}\text { Non-survivor }(n= \\
\text { 21) }\end{array}$ & Survivor $(n=10)$ & $\begin{array}{l}\mathbf{p} \\
\text { value }\end{array}$ \\
\hline$\geq 0.25$ to $<0.5$ & $3(10 \%)$ & $1(5 \%)$ & $2(20 \%)$ & .. \\
\hline$\geq 0.5$ to $<2.0$ & $16(52 \%)$ & $12(57 \%)$ & $4(40 \%)$ & .. \\
\hline$\geq 2.0$ & $8(26 \%)$ & $6(29 \%)$ & $2(20 \%)$ & .. \\
\hline \multicolumn{5}{|l|}{ Etiological findings } \\
\hline Gram-negative bacteria & $24(77 \%)$ & $19(90 \%)$ & $5(50 \%)$ & 0.022 \\
\hline CRGNB & $22(71 \%)$ & $18(86 \%)$ & $4(40 \%)$ & 0.015 \\
\hline CRKP & $15(48 \%)$ & $11(52 \%)$ & $4(40 \%)$ & 0.704 \\
\hline CRAB & $7(23 \%)$ & $7(33 \%)$ & 0 & 0.066 \\
\hline Gram-positive bacteria & $7(23 \%)$ & $3(14 \%)$ & $4(40 \%)$ & 0.170 \\
\hline MDR Gram-positive bacteria & $7(23 \%)$ & $3(14 \%)$ & $4(40 \%)$ & 0.170 \\
\hline MRSA & $1(3 \%)$ & $1(5 \%)$ & 0 & 1.000 \\
\hline MRCNS & $3(10 \%)$ & $1(5 \%)$ & $2(20 \%)$ & 0.237 \\
\hline E. faecium & $3(10 \%)$ & $1(5 \%)$ & $2(20 \%)$ & 0.237 \\
\hline Fungus & $1(3 \%)$ & 0 & $1(10 \%)$ & .. \\
\hline Same bacteria in SBI and lung infection & $20(65 \%)$ & $16(76 \%)$ & $4(40 \%)$ & 0.051 \\
\hline $\begin{array}{l}\text { Time from lung infection to SBI onset with the same } \\
\text { bacteria, days }\end{array}$ & $4.2(1.0 \sim 23.0)$ & $4.7(1.0 \sim 23.0)$ & $3.7(3.0 \sim 7.0)$ & 0.963 \\
\hline ICU admission prior to SBI onset & $22(71 \%)$ & $17(81 \%)$ & $5(50 \%)$ & 0.105 \\
\hline Invasive mechanical ventilation prior to SBI onset & $25(81 \%)$ & $18(86 \%)$ & $7(70 \%)$ & 0.358 \\
\hline Central venous catheter prior to SBI onset & $27(87 \%)$ & $19(91 \%)$ & $8(80 \%)$ & 0.577 \\
\hline Time from hospital admission to SBI onset, days & $22.0(2.0 \sim 64.0)$ & $23.0(2.0 \sim 64.0)$ & $18.0(2.0 \sim 41.0)$ & 0.393 \\
\hline Time from ICU admission to SBI onset, days & $14.0(2.0 \sim 30.0)$ & $13.0(2.0 \sim 30.0)$ & $8.0(7.0 \sim 27.0)$ & 0.187 \\
\hline $\begin{array}{l}\text { Time from invasive mechanical ventilation to SBI onset, } \\
\text { days }\end{array}$ & $12.0(2.0 \sim 30.0)$ & $13.0(2.0 \sim 30.0)$ & $10.5(6.0 \sim 28.0)$ & 0.696 \\
\hline Time from central venous catheter to SBI onset, days & $12.0(2.0 \sim 30.0)$ & $12.0(2.0 \sim 30.0)$ & $13.0(6.0 \sim 27.0)$ & 0.360 \\
\hline
\end{tabular}

For the laboratory findings, patients with SBI had elevated white blood cell counts, $\mathrm{C}$ reactive protein, and procalcitonin. The white blood cell count was increased in $22(80 \%)$ patients and decreased in $4(13 \%)$ patients. C-reactive protein was elevated in the majority of the patients and more than half of the patients had a value exceeding $100 \mathrm{mg} / \mathrm{L}$. All the 31 patients had elevated levels of procalcitonin (procalcitonin $\geq 0.1 \mathrm{ng} / \mathrm{mL}$ ). A total of 24 patients $(77 \%)$ demonstrated procalcitonin levels higher than $0.5 \mathrm{ng} / \mathrm{mL}$, whereas in 8 patients $(26 \%)$ this marker was higher than $2 \mathrm{ng} / \mathrm{mL}(26 \%)$.

Gram-negative bacteria were the main pathogens of SBIs. Compared with the survivors, the non-survivors were more likely to suffer from SBIs caused by carbapenem-resistant gram-negative bacteria (CRGNB). The most common pathogens were carbapenem-resistant Klebsiella pneumoniae (CRKP) and carbapenem-resistant Acinetobacter baumannii (CRAB), with detection rates of 48\% (15/31) and 23\% (7/31), respectively. Within 30 days following SBI onset, 11 (73\%) patients who were infected with CRKP did not survive. The same result was noted for 7 patients with CRAB. One case each was detected with MDR Acinetobacter baumannii (MDRAB) and extended-spectrum beta-lactamase (ESBL)-producing Klebsiella pneumoniae. The patient who was infected with MDRAB did not survive. Gram-positive bacteria were identified in 7 patients, including S. aureus, coagulase-negative Staphylococcus (CNS) and Enterococcus faecium (E. faecium). More than half of the patients were discharged. Candida parapsilosis was identified in one blood culture of a critically ill patient with COVID-19. 
Prior to the SBI onset, 22 (71\%) patients were admitted to the intensive care unit (ICU). A total of 25 (81\%) patients were treated with tracheal intubation and 27 (87\%) with central venous catheterization. The median durations from ICU admission, invasive mechanical ventilation, and central venous catheter to SBI onset were $14.0(2.0 \sim 30.0), 12.0(2.0 \sim 30.0)$ and 12.0 days $(2.0 \sim 30.0)$, respectively.

All patients were treated with a combination regimen of antibacterial drugs during the initial treatment (Table 3). A total of 20 (65\%) patients were treated with the carbapenems-based antibacterial regimen and the colistin-based regimen was used in 16 (52\%) patients. In order to cover infections that may be caused by gram-positive bacteria, 15 (48\%), the patients were treated with glycopeptides or linezolid. In 21 (68\%) patients, antifungal therapy was also implemented. However, these empirical treatments did not significantly improve the survival of these patients. 
Table 3

Treatments and outcomes of patients at SBI onset

\begin{tabular}{|c|c|c|c|c|}
\hline & Total $(n=31)$ & Non-survivor $(n=21)$ & Survivor $(n=10)$ & $\mathrm{p}$ value \\
\hline \multicolumn{5}{|l|}{ Initial empiric treatment } \\
\hline Carbapenems & $20(65 \%)$ & $15(71 \%)$ & $5(50 \%)$ & 0.423 \\
\hline Tigecycline & $7(23 \%)$ & $5(24 \%)$ & $2(20 \%)$ & 1.000 \\
\hline Colistin & $16(52 \%)$ & $12(57 \%)$ & $4(40 \%)$ & 0.458 \\
\hline Ceftazidime/avibatan & $5(16 \%)$ & $4(19 \%)$ & $1(10 \%)$ & 1.000 \\
\hline Glycopeptides or Linezolid & $15(48 \%)$ & $10(48 \%)$ & $5(50 \%)$ & 1.000 \\
\hline Antifungal treatment & $21(68 \%)$ & $14(67 \%)$ & $7(70 \%)$ & 1.000 \\
\hline Only 1 sensitive antimicrobial treatment & $16(52 \%)$ & $9(43 \%)$ & $7(70 \%)$ & 0.252 \\
\hline$\geq 2$ sensitive antimicrobial treatment & $7(23 \%)$ & $5(24 \%)$ & $2(20 \%)$ & 1.000 \\
\hline \multicolumn{5}{|l|}{ Targeted treatment } \\
\hline CRKP & $12 / 12(100 \%)$ & $8 / 12(67 \%)$ & $4 / 12(33 \%)$ & $\ldots$ \\
\hline Carbapenems + colistin & $3 / 12(25 \%)$ & $3 / 12(25 \%)$ & 0 & 0.533 \\
\hline Carbapenems + Tigecycline & $2 / 12(17 \%)$ & $2 / 12(17 \%)$ & 0 & 1.000 \\
\hline Tigecycline + colistin & $1 / 12(8 \%)$ & $1 / 12(8 \%)$ & 0 & 1.000 \\
\hline Tigecycline + Ceftazidime/avibatan & $2 / 12(17 \%)$ & 0 & $2 / 12(17 \%)$ & 0.097 \\
\hline Colistin + Ceftazidime/avibatan & $2 / 12(17 \%)$ & $1 / 12(8 \%)$ & $1 / 12(8 \%)$ & 1.000 \\
\hline Colistin + Ceftazidime/avibatan + Sulbactam preparations & $1 / 12(8 \%)$ & 0 & $1 / 12(8 \%)$ & 0.323 \\
\hline Colistin + Ceftazidime/avibatan + Tigecycline & $1 / 12(8 \%)$ & $1 / 12(8 \%)$ & 0 & 1.000 \\
\hline CRAB & $4 / 4(100 \%)$ & $4 / 4(100 \%)$ & 0 & $\ldots$ \\
\hline Carbapenems + colistin & $1 / 4(25 \%)$ & $1 / 4(25 \%)$ & 0 & 1.000 \\
\hline Tigecycline + colistin & $1 / 4(25 \%)$ & $1 / 4(25 \%)$ & 0 & 1.000 \\
\hline Tigecycline + Sulbactam preparations & $2 / 4(50 \%)$ & $2 / 4(50 \%)$ & 0 & 1.000 \\
\hline \multicolumn{5}{|l|}{ Outcomes } \\
\hline Sepsis & $28(90 \%)$ & $21(100 \%)$ & $7(70 \%)$ & 0.027 \\
\hline Septic shock & $23(74 \%)$ & $19(90 \%)$ & $4(40 \%)$ & 0.006 \\
\hline Coagulopathy & $24(77 \%)$ & $18(86 \%)$ & $6(60 \%)$ & 0.172 \\
\hline Acute cardiac injury & $24(77 \%)$ & $19(90 \%)$ & $5(50 \%)$ & 0.022 \\
\hline Acute liver injury & $20(65 \%)$ & $14(67 \%)$ & $6(60 \%)$ & 1.000 \\
\hline Acute kidney injury & $14(45 \%)$ & $10(48 \%)$ & $4(40 \%)$ & 1.000 \\
\hline Hospital mortality & $24(77 \%)$ & $21(100 \%)$ & $3(30 \%)$ & \\
\hline Hospital length of stay, days & $32.0(12.0-80.0)$ & $29.0(12.0 \sim 66.0)$ & $52.0(13.0 \sim 80.0)$ & 0.001 \\
\hline Time from SBI onset to sepsis, days & $1.0(1.0 \sim 5.0)$ & $1.0(1.0 \sim 5.0)$ & $1.0(1.0 \sim 3.0)$ & 0.959 \\
\hline Time from SBI onset to septic shock, days & $3.0(1.0 \sim 12.0)$ & $3.0(1.0 \sim 12.0)$ & $2.0(1.0 \sim 4.0)$ & 0.409 \\
\hline Time from SBI onset to death or discharge, days & $8.0(1.0 \sim 76.0)$ & $7.0(1.0 \sim 14.0)$ & $40.0(6.0 \sim 76.0)$ & $<0.0001$ \\
\hline
\end{tabular}


Among the patients infected by CRKP and CRAB, 6 patients died quickly within 2 days following the onset of SBI, failing to implement targeted treatment. The other 12 patients infected by CRKP were provided 7 different regimens, of which carbapenems combined with colistin regimen was the most commonly used. In the targeted treatment of CRAB, the regimen of tigecycline combined with sulbactam preparations was the most commonly used. However, the patients did not survive.

Once bloodstream infection occurred, the condition of the majority of the patients progressed very rapidly. The median score of SOFA at SBI onset was 8.0 (2.0-19.0) (Table 3). Almost every patient with SBI had complication due to sepsis (28 [90\%]) and the second most common complications included coagulopathy (24 [77\%]), acute cardiac injury (24 [77\%]), septic shock (23 [74\%]) and acute liver injury (20 [65\%]). The median time to sepsis and septic shock were 1.0 days $(1.0 \sim 5.0)$ and 3.0 days $(1.0 \sim 12.0)$, respectively.

The proportion of CRGNB detected in the blood cultures of non-survivors was higher (18 [86\%] vs. 4 [40\%]) compared with that of the survivors, and these subjects were more likely to develop sepsis (21 [100\%] vs. 7 [70\%]) and acute cardiac injury (19 [90\%] vs. 5 [50\%]).

\section{Discussion}

The present study reported 31 patients with SBI during hospitalization for COVID-19 treatment, with an incidence rate of 1.9\% and a 30-day high mortality of $67.7 \%$. In this retrospective study, the clinical features, laboratory findings, treatments, and outcomes of the patients with SBI were described. Increased risk of death was noted for the following three subgroups: patients infected with CRGNB, patients with complications of sepsis and patients with acute cardiac injury.

The mortality of hospitalized patients with COVID-19 is high and SBI is one of the important causes of their deaths.[5] For bloodstream infections, optimized management strategies include early diagnosis, appropriate empiric and targeted antibacterial therapy and prompt effective source control.[19] However, in previously published studies,[7, 8, 10, 11] only the etiology and the risk factors of SBI have been reported. The clinical features, treatment measures and disease progression of patients with the onset of SBI have not been previously reported. To the best of our knowledge, the present study is the first to review the clinical course of SBI with COVID-19.

Following calculation of the incidence of SBI, patients with CNS were excluded in these blood cultures. These patients had either no significant changes in clinical features, or the pathogens were isolated only once per patient, which was considered a skin symbiotic bacterial contamination. The incidence of SBI reported in the present study was consistent with the strictly calculated incidences reported in New York City and London, which were $1.6 \%$ [8]and $2.5 \%$ [10], respectively.

In the present study, the 30-day mortality following SBI onset and the hospital mortality were higher than the mortality of severe ill patients of COVID-19.[3] These indices were also higher than the mortality (50\%) of SBI in Copenhagen University Hospital.[7] This outcome is probably related to $71 \%$ of SBI cases in the study, which were caused by CRKP or CRAB. A higher distribution was noted in the non-survivors. According to these reports, $[20,21]$ the mortality rate of patients with bloodstream infection caused by CRKP was as high as $41.6 \% \sim 58.7 \%$. The mortality rate of bloodstream infections caused by CRAB was also very high, reaching 54.7\%.[22] Bloodstream infection itself is an event with an extreme impact on mortality. In addition, it occurs during the treatment of COVID-19, which may increase the risk of death. The detection of CRGNB is one of the risk factors for patient death following bloodstream infections.[23-26] The findings confirmed that the nonsurvivors had higher CRGNB distribution. It was speculated that in SBI patients with COVID-19, CRGNB also affected the outcomes of the patients.

Although most patients with SBI indicated fever at the time of onset, other patients did not show this symptom. Almost all patients did not present with chills or fear of cold. This led to the conclusion that the difficulty of clinical diagnosis of SBI was increased. The clinical diagnosis of SBI requires a comprehensive judgment based on changes in the patient temperature, breathing, blood pressure and state of consciousness.

The current research demonstrated that conventional infection indicators, such as procalcitonin, C-reactive protein and white blood cell counts were elevated during SBI onset, which strongly supported the judgment of bacterial infections and enabled clinicians to administer antimicrobials/antibacterial agents in time. In some patients, procalcitonin and C-reactive protein exceeded $2 \mathrm{ng} / \mathrm{mL}$ and $100 \mathrm{mg} / \mathrm{L}$, respectively, indicating the severity of the infections and suggesting that more active treatment should have been provided.

All patients in the SBI group were treated with empirical combined application of antibacterial drugs and the majority of the treatment options were based on carbapenems, combined with glycopeptides or linezolid to treat possible positive bacterial infection. However, this approach did not show obvious benefits, which was related to the fact that most of the pathogens were carbapenem-resistant bacteria. Patients with previous antibacterial use, mechanical ventilation and previous ICU stay were susceptible to carbapenem-resistant bacteria.[27] For such patients, the risk of drug resistance should be fully considered. The application of carbapenems may be ineffective. The present 
study demonstrated that in empirical treatment, timely administration of sensitive antibacterial drugs exhibited no obvious benefit in improving the mortality of the patients. We speculated that the potentially serious condition of the patients exhibited a more important impact on mortality and may mask the influence of antibacterial therapy.[28]

Although several reports have been performed on the treatment of CRKP bloodstream infections, it is not certain whether it is applicable to patients with COVID-19. In the targeted treatment stage, seven programs were applied in 12 patients with CRKP SBI, revealing no clinically effective treatment program. The combination regimen based on ceftazidime/avibactam may be more effective. $71 \%(5 / 7)$ of the patients survived within 30 days. It is also worth mentioning that one patient was treated with ceftazidime/avibactam combined with colistin and sulbactam preparations and his condition was finally improved leading to his discharge. Previous studies have reported that ceftazidime/avibactam is an effective treatment for CRKP bloodstream infections, which can significantly reduce the mortality of the patients.[29-31] The effectiveness of the ceftazidime/avibactam-based regimen in the treatment of CRKP bloodstream infections secondary to COVID-19 requires further investigation.

In a study by Xu and colleagues,[32] sulbactam-based treatment options may be more effective for CRAB bloodstream infections. In the present study, 4 patients used 3 regimens, including carbapenem plus colistin, tigecycline plus colistin and tigecycline plus sulbactam preparations (2/4). Unfortunately, they all failed. The risk of CRAB bloodstream infections secondary to COVID-19 was more serious.

The present study demonstrated that patients, who belonged in the non-survivor group, were more likely to have complications, such as sepsis and acute cardiac injury following SBI onset. The essence of sepsis involves a life-threatening organ dysfunction caused by a dysregulated host in response to infection.[14,33] If timely and effective treatment is not implemented, sepsis is fatal. The majority of the pathogens of SBIs with COVID-19 are CRGNB, which are difficult to treat and more difficult to result in successful treatment. Therefore, when sepsis occurs, the risk of death increases.

As identified in previous studies, acute cardiac injury is a common complication of sepsis and the resulting cardiac dysfunction is also one of the main causes of death in patients with sepsis.[34,35] Acute cardiac injury is the most reported cardiac abnormality in COVID-19 and is strongly associated with mortality.[36, 37] Prior to SBI onset, several patients experienced acute cardiac injury. When SBI occurs, the patient cardiac injury aggravates and the risk of death increases.

The present study contains certain limitations. First, the number of samples of SBls was small, which could not fully reveal the risk factors for the mortality of SBIs. Secondly, this was a single-center retrospective study. Further multi-center studies and prospective research data are required to explore the optimal treatments for SBI with COVID-19. Finally, this designated hospital is specialized in treating mainly patients with severe or critically ill COVID-19. Therefore, the incidence of SBIs may be higher than the incidence of the entire COVID-19.

In conclusion, this retrospective study indicated that SBIs with COVID-19 were associated with high 30-day mortality. The survival time of the non-survivors was likely to be within 7 days following SBI onset. Increased risk of death was noted for the following three subgroups: patients infected with CRGNB, patients with complications of sepsis and patients with acute cardiac injury.

\section{Abbreviations}

COVID-19: corona virus disease 2019; SARS-CoV-2: severe acute respiratory syndrome coronavirus 2; SBI: secondary bloodstream infection; SOFA: Sequential Organ Failure Assessment; MDR: Multidrug-resistant; ECDC: European Centre for Disease Prevention and Control; CDC: Centers for Disease Control and Prevention; CCl: Charlson Comorbidity Index; CRGNB: carbapenem-resistant gram-negative bacteria; CRKP: carbapenem-resistant Klebsiella pneumoniae, CRAB: carbapenem-resistant Acinetobacter baumannir, MDRAB: MDR Acinetobacter baumannii; ESBL: extended-spectrum beta-lactamase; CNS: coagulase-negative Staphylococcus; E. faecium: Enterococcus faecium; $S$. aureus: Staphylococcus aureus.

\section{Declarations}

\section{Acknowledgments}

We acknowledge the supports from colleagues in Wuhan Union Hospital in the interpretation about the microbiological results.

\section{Informed consent}

Not applicable.

\section{Authors' contributions}


$\mathrm{XC}, \mathrm{SW}$, and $\mathrm{YZ}$ designed the study and had full access to all of the data in the study. They were also responsible for the integrity of the data and the accuracy of the data analysis. JL, JW, and YY drafted the manuscript. JL, JW, YY, JC, and PC collected, analyzed and interpreted the clinical and laboratory data. JW and JL performed data analysis. The author(s) read and approved the final version of the manuscript.

\section{Funding}

Not applicable.

\section{Availability of data and materials}

The supporting data are available with the corresponding author and laboratory depositories.

\section{Ethics approval and consent to participate}

The study was approved by the Ethics Committee of Union Hospital, Tongji Medical College, Huazhong University of Science and Technology (Permission number: [2020]0104).

\section{Consent for publication}

The researchers have full right to publish the findings.

\section{Competing Interests}

We declare that we have no conflicts of interest.

\section{References}

1. Callaway E, Ledford H, Viglione G, Watson T, Witze A. COVID and 2020: An extraordinary year for science. Nature. 2020; 588:550-2.

2. World Health Organization. WHO Coronavirus Disease (COVID-19) Dashboard. Available at: https://covid19.who.int. Accessed 4/27 2020.

3. COVID-19 Treatment Guidelines Panel. Coronavirus Disease 2019 (COVID-19) Treatment Guidelines. Available at: https://www.covid19treatmentguidelines.nih.gov/. Accessed 4/21 2020.

4. Yang $X, Y u$ Y, Xu J, et al. Clinical course and outcomes of critically ill patients with SARS-CoV-2 pneumonia in Wuhan, China: a singlecentered, retrospective, observational study. Lancet Respir Med. 2020; 8:475-81.

5. Zhou F, Yu T, Du R, et al. Clinical course and risk factors for mortality of adult inpatients with COVID-19 in Wuhan, China: a retrospective cohort study. Lancet. 2020; 395:1054-62.

6. Huang C, Wang Y, Li X, et al. Clinical features of patients infected with 2019 novel coronavirus in Wuhan, China. Lancet. 2020; 395:497506.

7. Engsbro AL, Israelsen SB, Pedersen M, et al. Predominance of hospital-acquired bloodstream infection in patients with Covid-19 pneumonia. Infect Dis (Lond). 2020; 52:919-22.

8. Sepulveda J, Westblade LF, Whittier S, et al. Bacteremia and Blood Culture Utilization during COVID-19 Surge in New York City. J Clin Microbiol. 2020; 58.

9. Goyal P, Choi JJ, Pinheiro LC, et al. Clinical Characteristics of Covid-19 in New York City. N Engl J Med. 2020; 382:2372-4.

10. Hughes S, Troise O, Donaldson H, Mughal N, Moore LSP. Bacterial and fungal coinfection among hospitalized patients with COVID-19: a retrospective cohort study in a UK secondary-care setting. Clin Microbiol Infect. 2020; 26:1395-9.

11. Li J, Wang J, Yang Y, et al. Etiology and antimicrobial resistance of secondary bacterial infections in patients hospitalized with COVID-19 in Wuhan, China: a retrospective analysis. Antimicrob Resist Infect Control. 2020; 9:153.

12. National Health Commission of the People's Republic of China. New coronavirus pneumonia prevention and control program (7th ed) (in Chinese). Available at:

http://www.nhc.gov.cn/yzygj/s7653p/202003/46c9294a7dfe4cef80dc7f5912eb1989/files/ce3e6945832a438eaae415350a8ce964.pdf. Accessed 1/20 2021.

13. Horan TC, Andrus M, Dudeck MA. CDC/NHSN surveillance definition of health care-associated infection and criteria for specific types of infections in the acute care setting. Am J Infect Control. 2008; 36:309-32.

14. Singer M, Deutschman CS, Seymour CW, et al. The Third International Consensus Definitions for Sepsis and Septic Shock (Sepsis-3). JAMA. 2016; 315:801-10. 
15. Magiorakos AP, Srinivasan A, Carey RB, et al. Multidrug-resistant, extensively drug-resistant and pandrug-resistant bacteria: an international expert proposal for interim standard definitions for acquired resistance. Clin Microbiol Infect. 2012; 18:268-81.

16. Zhang Y, Guo L, Song W, Wang Y, Dong F, Liu G. Risk factors for carbapenem-resistant K. pneumoniae bloodstream infection and predictors of mortality in Chinese paediatric patients. BMC infectious diseases. 2018; 18:248.

17. Kdigo KD, Outcomes IG. Acute Kidney Injury Work Group: KDIGO clinical practice guideline for acute kidney injury. Kidney international Supplement. 2012; 2:1-138.

18. McCarthy C, Raber I, Chapman A, et al. Myocardial Injury in the Era of High-Sensitivity Cardiac Troponin Assays: A Practical Approach for Clinicians. JAMA cardiology. 2019; 4:1034-42.

19. Giannella M, Bartoletti M, Gatti M, Viale P. Advances in the therapy of bacterial bloodstream infections. Clinical microbiology and infection: the official publication of the European Society of Clinical Microbiology and Infectious Diseases. 2020; 26:158-67.

20. Chang H, Wei J, Zhou W, et al. Risk factors and mortality for patients with Bloodstream infections of Klebsiella pneumoniae during 20142018: Clinical impact of carbapenem resistance in a large tertiary hospital of China. Journal of infection and public health. 2020; 13:78490.

21. Tumbarello M, Viale P, Viscoli C, et al. Predictors of mortality in bloodstream infections caused by Klebsiella pneumoniae carbapenemase-producing K. pneumoniae: importance of combination therapy. Clinical infectious diseases: an official publication of the Infectious Diseases Society of America. 2012; 55:943-50.

22. Balkhair A, Al-Muharrmi Z, Al'Adawi B, et al. Prevalence and 30-day all-cause mortality of carbapenem-and colistin-resistant bacteraemia caused by Acinetobacter baumannii, Pseudomonas aeruginosa, and Klebsiella pneumoniae: Description of a decade-long trend. International journal of infectious diseases: IJID: official publication of the International Society for Infectious Diseases. 2019; 85:10-5.

23. Santoro A, Franceschini E, Meschiari M, et al. Epidemiology and Risk Factors Associated with Mortality in Consecutive Patients with Bacterial Bloodstream Infection: Impact of MDR and XDR Bacteria. Open forum infectious diseases. 2020; 7: 461.

24. Giannella M, Pascale R, Pancaldi L, et al. Follow-up blood cultures are associated with improved outcome of patients with gram-negative bloodstream infections: retrospective observational cohort study. Clinical microbiology and infection: the official publication of the European Society of Clinical Microbiology and Infectious Diseases. 2020; 26:897-903.

25. Huh K, Chung D, Ha Y, et al. Impact of Difficult-to-Treat Resistance in Gram-negative Bacteremia on Mortality: Retrospective Analysis of Nationwide Surveillance Data. Clinical infectious diseases: an official publication of the Infectious Diseases Society of America. 2020; 71: e487-e96.

26. Xiao T, Zhu Y, Zhang S, et al. A Retrospective Analysis of Risk Factors and Outcomes of Carbapenem-Resistant Klebsiella pneumoniae Bacteremia in Nontransplant Patients. The Journal of infectious diseases. 2020; 221: S174-S83.

27. Palacios-Baena Z, Giannella M, Manissero D, et al. Risk factors for carbapenem-resistant Gram-negative bacterial infections: a systematic review. Clinical microbiology and infection: the official publication of the European Society of Clinical Microbiology and Infectious Diseases. 2020.

28. Gutiérrez-Gutiérrez B, Salamanca E, de Cueto M, et al. Effect of appropriate combination therapy on mortality of patients with bloodstream infections due to carbapenemase-producing Enterobacteriaceae (INCREMENT): a retrospective cohort study. The Lancet Infectious diseases. 2017; 17:726-34.

29. Karaiskos I, Daikos G, Gkoufa A, et al. Ceftazidime/avibactam in the era of carbapenemase-producing Klebsiella pneumoniae: experience from a national registry study. The Journal of antimicrobial chemotherapy. 2020.

30. Wu G, Abraham T, Lee S. Ceftazidime-Avibactam for Treatment of Carbapenem-Resistant Enterobacteriaceae Bacteremia. Clinical infectious diseases: an official publication of the Infectious Diseases Society of America. 2016; 63:1147-8.

31. Jorgensen S, Trinh T, Zasowski E, et al. Real-World Experience with Ceftazidime-Avibactam for Multidrug-Resistant Gram-Negative Bacterial Infections. Open forum infectious diseases. 2019; 6: 522.

32. Xu S, Li Y, Xu X, et al. A Case-Control Study: Clinical Characteristics of Nosocomial Bloodstream Infections Versus Non-bloodstream Infections of Acinetobacter spp. Clinical infectious diseases: an official publication of the Infectious Diseases Society of America. 2018; 67: S189-S95.

33. Rhodes A, Evans L, Alhazzani W, et al. Surviving Sepsis Campaign: International Guidelines for Management of Sepsis and Septic Shock: 2016. Intensive care medicine. 2017; 43:304-77.

34. Romero-Bermejo F, Ruiz-Bailen M, Gil-Cebrian J, Huertos-Ranchal M. Sepsis-induced cardiomyopathy. Current cardiology reviews. 2011; 7:163-83.

35. Sanfilippo F, Corredor C, Fletcher N, et al. Diastolic dysfunction and mortality in septic patients: a systematic review and meta-analysis. Intensive care medicine. 2015; 41:1004-13. 
36. Dou Q, Wei X, Zhou K, Yang S, Jia P. Cardiovascular Manifestations and Mechanisms in Patients with COVID-19. Trends in endocrinology and metabolism: TEM. 2020; 31:893-904.

37. Wei Z, Geng Y, Huang J, Qian H. Pathogenesis and management of myocardial injury in coronavirus disease 2019. European journal of heart failure. 2020; 22:1994-2006. 